\title{
An approach to chronic pain of non-malignant origin
}

\author{
B. L. CRUE* \\ M.D., F.A.C.S.
}

J. J. PINSKY†

M.D.

*Department of Neurological Surgery, University of Southern California School of Medicine, Los Angeles, and New Hope Pain Center and †New Hope Pain Research Foundation, Pasadena, California, U.S.A.

\section{Introduction}

There is no such thing as a pain stimulus in nature. Only tissue damage, or, in the intact integument, mechanical displacement or thermal change (Kenton, Crue and Carregal, 1975, 1976) is adequate to initiate primary sensations. There is no place for pain to be classified as a primary sensation (Crue and Carregal, 1975; Crue et al., 1976). Acute pain is better considered as a perception; the related physiological mechanisms are largely within the central nervous system itself, and require a consciously functioning brain for the subjective experience of pain. The peripheral causative input mechanism necessary to initiate the perception of pain has been labelled as nociception, and there is some specificity in the human peripheral nervous system, with high threshold thermal receptors and high threshold mechanoreceptors (called nociceptors) both capable of initiating peripheral nociceptive input. These nociceptors, along with chemoreceptors, seem to be activated in most cases by acute injury and tissue damage to transmit the very slow $\mathrm{C}$ fibre and faster A delta input system that constitute only a small part of an overall patterning of input. There are both peripheral and central nervous system spatial overlap factors, convergence, temporal summation, and amplitude intensity factors that then give rise to the central perceptual event of pain. In the human, this pain 'begins' in the region of the dorsal horn of the spinal cord (Crue and Carregal, 1975). We still do not know enough about the patterning of input into the spinal cord dorsal horn region; but, apparently, pressure, temperature change, or tissue damage can, if the pattern is correct, lead to message transmission centrally, that comes to be perceived in consciousness as pain (Fig. 1). However, it should be noted that this general mode of patterned inputs could also be involved in non-noxious stimulus transmission that can also be perceived in consciousness as pain (vide infra).

A classification of pain (Agnew, Crue and Pinsky, 1979; Crue and Pinsky, 1984; Crue et al., 1979) based on the length of time that pain has been present is 3 . shown in Table 1 .

\section{Acute pain}

From a neurophysiological standpoint it would ${ }_{\infty}$ appear that acute pain in the human implies tissue damage with subsequent sequential afferent nocicep- tive input. The acute pain generally responds well to the natural healing process, often with treatment, by repair, removal, or neutralization of the original $\frac{\mathbb{}}{3}$ aetiological source. There is, however, no good evidence for the presence of continuing active tissoe injury or disease in the chronic non-cancer pain problems on which we will focus our attention.

In normal use by many patients and physicians, the phrase 'acute pain' is often extended to include reference to an amplitude or intensity factor. It is as if $\frac{}{\circ}$ an acute pain is synonymous with a severe pain. This common usage creates problems because it implies or $\stackrel{\square}{\square}$ relegates attributes to the pain that are the bases for $\overrightarrow{\vec{F}}$ the formation of conceptual errors about the pain 3 experience. Acute pain should only mean pain that has not gone on for very long, and it can range from? mild to severe in intensity and seriousness.

\section{Chronic pain}

To discuss chronic pain, we must decide on the temporal difference that divides chronic from acute pain. We indicate a period after which the pain is no longer generally considered acute, or even subacute? (days to weeks usually). Empirically, Sternbach $\frac{7}{0}$ (1974) has suggested the cut-off time of 6 months. While this may be a valid statistical designation, $\mathbb{N}$ bona fide chronic pain syndromes can be seen earlier than 6 months, particularly following an acute event. N

One then wonders whether all chronic pain must $\omega$ have arisen originally as acute pain, and only been? called chronic after the passage of time. This woulde be true if chronic pain were the same physiological entity as acute pain. It is at that point that we feel ${ }^{+}$ there is a major obstacle in the understanding of 


\begin{tabular}{c}
\hline Afferent input \\
of \\
spinal dorsal root
\end{tabular}

(1) Cutaneous

(a) Touch $\rightarrow$ Discriminative - fast adapting and fast conducting (Hair nets and Meisner corpuscles) and some slowly adapting but fast conducting type II mechanoreceptors. Up ipsilateral dorsal column with some branches to dorsal horn + to ventral horn for motor reflex

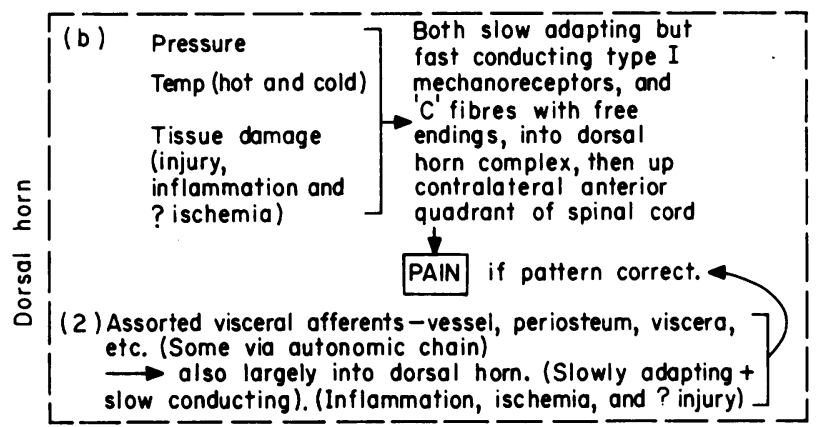

(3) Proprioception portion of grovity sense, largely 'nonadapting and fast conducting, up ipsilateral dorsol column to higher CMS (including consciousness) with branches to dorsal born + ventral born for gamma loop plus for motor reflex + spinocerebellar tracts for equilibrium (subconscious)

FIG. 1. The patterning of the input of the spinal cord dorsal horn region.

chronic pain. What all too commonly occurs is that chronic pain is referred to and explained as though it were a direct continuation of acute pain with continuing constant nociceptive input from an injured area. The quasi-explanations of continuing pathophysiology in the periphery include: it [the lesion] has failed to heal,' 'it [the injured area] was not fixed right' (usually by another surgeon). When there have been numerous surgical attempts to quell the pain, a frequent explanation that is given is that 'the pain is from the scar remaining after the prior surgery'. There is no evidence that most people with chronic pain have scarred in a different anatomical pattern or with an altered physiological responsiveness when compared to people without chronic pain who have had the same history of tissue damage and reparative efforts. Patients with chronic pain have not been identified as having any unusual characteristics of tissue healing.

Chronic pain that continues as more or less constant pain should be regarded as a central pain, without any need to invoke the presence of intermittent events or continued peripheral nociceptive input. This may be regarded as a tautological classification, but the absence of a source of peripheral nociceptive input is much more the rule than the exception when there is chronic pain of a more or less constant nature. If a pain problem is accompanied by clear evidence of a source of nociceptive input in the periphery, we would not classify it as chronic pain (Crue et al., 1980). The best available evidence suggests that there is no need for any classification of chronic benign (non-neoplastic) pain with continuing nociceptive input. The classification of chronic pain in Table 1 is based on the acceptance of the assumptions that the maintenance of chronic pain chronically is due only to central factors (or generators) in the absence of continued peripheral nociceptive input.

When there is chronic pain as we have defined it, the idea that there is continued nonciceptive stimulation from the periphery as its cause is not supported by fact. Nonetheless, nociceptive input continues to be used commonly as reason for the constant pain even when its somatic source(s) can rarely be definitively isolated. It is necessary that these relationships be grasped so that treatment can be linked to the syndrome's existing aetiological and perpetuating factors, and not to undemonstrable hypothesized pathophysiological mechanisms. At this juncture, we 
TABLE 1. Temporal classification of pain complaints

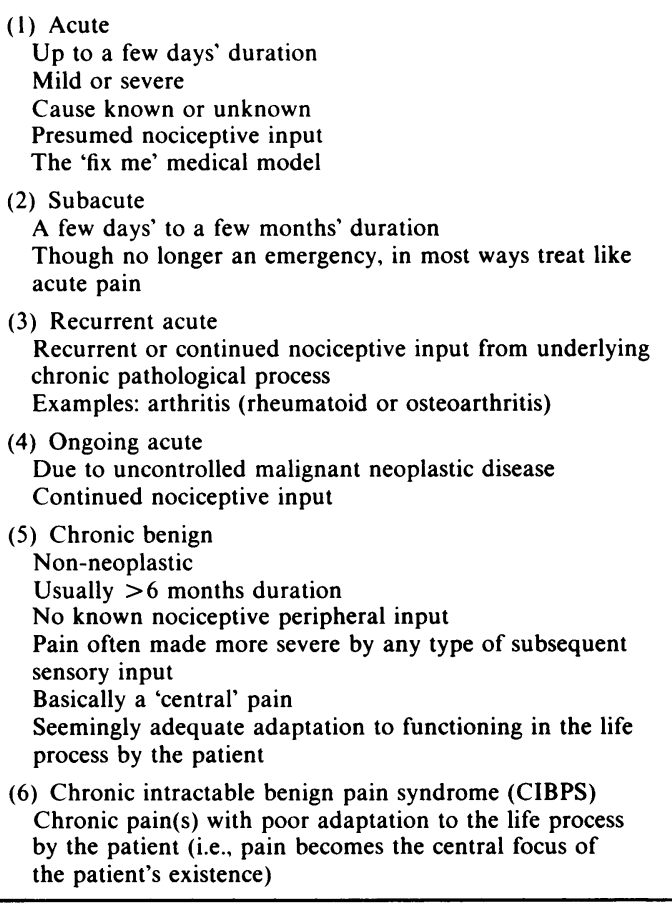

can begin to see the necessity for acceptable working definitions and classification, because they affect how we form our concepts of the aetiology of acute and chronic pain from which people suffer, and this strongly affects the approach to treatment.

\section{Chronic pain syndromes}

\section{Peripheralist and centralist conceptualizations}

The peripheralist generally considers chronic pain in the same neurophysiological and organic medical models used for acute pain. A causal link is assumed between the chronic pain and continued nociceptive input that is presumed to be related to an active peripheral abnormality that continues to be a source of nociceptive stimuli. It is regarded for hypothetical reasons that the original causative injury or pathological condition has not healed adequately, or new trouble has started. This conceptual model is based on presumed continuing active tissue or anatomical abnormality, even when no abnormality is found with a thorough medical investigation. When noncancer related pain becomes the central feature of a chronic syndrome, what is conveniently not confronted by the peripheralist is the fact that many more patients with similar past injuries and treat- ments have had a decrease or disappearance of their 3 pain in spite of the omnipresent viscissitudes associ- $\stackrel{\varnothing}{\varrho}$

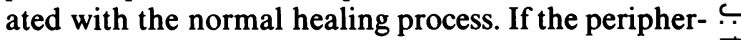
alist conceptual model is pursued for a problem of $\overrightarrow{\vec{F}}$ chronic pain, it then quite properly would follow that therapy would be centered around attempts 'to fix' the presumed remaining peripheral problem or to block the assumed continued nociceptive input, $\overparen{\curvearrowright}$ either by anaesthetic peripheral afferent nerve blocks $\circ$ (Kamdar, 1979) or by more central neurosurgical के sensory pathway blocking or ablative procedures $\vec{\circ}$ (Felsööry and Crue, 1976).

The centralist does not presume the presence of $\vec{\omega}$ continued peripheral nociceptive input as an explanation for chronic pain in most human chronic benign pain syndromes. In our experience, the peripheralist approach to chronic pain has resulted in too many surgical and pharmaceutical invasive $\unlhd$ treatments for these medically based chronic pain syndromes.

There are some apparent exceptions, and there are some chronic pain syndromes that seem to originate entirely within the central nervous system after specific organic lesions. Examples of this include $\mathbb{D}$ some infarcts of the thalamus (Dejerine-Roussy pain syndrome), and from pain after infarcts lower in the brainstem (Wallenberg syndrome with pain). How ever, even in these syndromes, it must be rement $\vec{\varphi}$ bered that most people who have these types of lesions do not present for medical treatment sufferine with chronic pain as part of their clinical picture (Agnew, 1984).

There is also general agreement that the central nervous system is able to pervert non-noxious light touch, pressure, thermal, or auditory sensory input so $\triangle$ that these stimuli act as though they were noxious $\overrightarrow{\overrightarrow{0}}$ and can trigger acute and severe paroxysms of pain located by the patient in the periphery and usually labelled as 'neuralgia' or 'neuralgic jabs' (Crue and Carregal, 1974; Crue and Saltzberg, 1979; Crue, Todd and Carregal, 1968). Examples of this are seen in multiple sclerosis, or in primary idiopathic trigeminal neuralgia, in which a light touch almost anywhere on the face can trigger paroxysms of severe 0 neuralgic jabs of pain in distributions of the fifth cranial nerve. The important point to remember is $\frac{9}{3}$ that these events represent a central perversion of $D$ non-noxious peripheral input. Also, there is the phenomenon of 'slop over' that is not fully under- N stood. It appears to apply in conditions in which there is close neuroanatomical intertwining between $\tilde{N}$ an area injured and one without injury. An example $\omega$ of this is the ulnar distribution arm pain with myocardial infarction related to a shared visceralo afferent input to the $T_{1}$ cord level by the myocardium $\overparen{D}$ and the ulnar nerve. None of these are examples of $\stackrel{\mathcal{P}}{+}$ spontaneous central pain states, and most neuralgic 7 
pain is spontaneous. This implies a central generator mechanism that, on occasion, without peripheral input as a trigger, can be the source of a pain experience that is perceived as being felt in, and 'coming from', some specific peripheral anatomic area, as part of the body image. Crue has long considered that such neuralgic pain can be considered a form of 'central sensory epilepsy', and must have an underlying neurophysiological central mechanism (Carregal, Crue and Todd, 1963; Crue, Carregal and Todd, 1964; Crue et al., 1967; Crue et al., 1956; Crue and Sutin, 1959; Crue, Todd and Carregal, 1970).

\section{Central neurological features}

Table 2 reviews central factors that might be operative in either initiating or maintaining such a central generator mechanism underlying chronic pain (Crue, 1979). At times there can be organic abnormalities within the brain itself. However, there may be no central pathophysiology as far as is known, and yet central mechanisms can become overwhelmed by abnormal patterns of altered peripheral input. This is assumed to be the mechanism underlying many cases in which there is a partial injury of a mixed motor and sensory peripheral nerve, or even a nerve root, that results in 'cross-talk'; as a result of this, there may be the establishment of a physiologically abnormal neuronal pool or network centrally. It is suspected that this situation leads to continuing uncontrolled neuronal discharge, both cephalad to consciousness and peripherally to abnormal reflex output mechanisms. This is the situation that is generally accepted to apply in causalgia and the deafferentiation and reflex sympathetic dystrophy syndromes. It appears that there is a combination of both above mechanisms in the so-called central deafferentiation states in which the pain syndrome seems to have been initiated by some peripherally-based pathophysiology. Trigeminal neuralgia is an example of this proposed mechanism. The question of the pathologically involved anatomical level(s) within the central nervous system in deafferentiation or causalgic pain states remains not fully settled. There is no question that some central pain syndromes may be considered to be based more strongly on organic features than the more frequent chronic pain syndromes (Table 2, no. 3 ) in which active organic features have essentially ceased to play a role.

There is no conclusive evidence about the presence of inherited or acquired characteristics in the neuroanatomical circuitry or the neurochemical generative systems of the brain related to hypersensitive perception of pain. Balances or imbalances of nervous
TABLE 2. Chronic pain-predisposing central factors

(1) Organic abnormality within the central nervous system

(a) Pathological (such as post-stroke)

(b) Physiological, from abnormal peripheral input

(such as causalgia)

(c) Mixture of pathological and physiological (such

as tic douloureux possibly)

(2) Possible genetic or acquired differences with respect to perception of pain

(3) Psychological factors with no known physiological abnormality

(a) Past input into memory storage, particularly including early life events and developmental pattern

(b) Life situational events that accompany acute pain, injury or illness that act as causative triggers and sustaining forces

(c) Mixture of both of these (the CIBPS syndrome)

system activity, viscissitudes of excitation or inhibition, or differences in any number of central nervous system transmitter substances have not as yet explained pain hypersensitivity (Table 2, no. 2). However, there is no doubt that the converse is true; and, there are families with congenital insensitivity to pain. However, these people's characteristics are present without any known neuroanatomic or neurophysiological basis. Most normal individual differences in sensitivity to acute pain, when studied, turn out to be idiosyncratic with some more or less cultural/learned patterns superimposed (Zborowski, 1969).

The dynamics of chronic pain syndromes in the central nervous system include the possibility that within the central nervous system there is some type of underlying central generator. The latter can be pictured as an epileptiform focus (foci), or as a specific and functionally interlacing neuronal network. The latter may be generally conceptualized as being within the limbic lobe system that feeds back to loci of the nociceptive input modulating mechanism, related to the endorphin and enkephalin systems. These neuromodulator systems may affect not only acutely occurring or continuing afferent nociceptive input ascending from below. There are no research findings to tell us with any certainty whether or not this exists or how it might work. But the patient with chronic pain continues, after the nociceptive input wanes, to perceive pain and suffer with it. We have long suggested (Crue et al., 1964; Crue and Todd, 1968; Crue et al., 1968) that the appropriate generic pain label for this group of patients is 'neuralgia'. Furthermore, we believe this type of mechanism can explain all chronic pain syndromes in categories 5 and 6 of the classification in Table 1. This type of chronic pain syndrome has been described by us in the past (Pinsky, 1978, 1983) as a chronic intractable benign pain syndrome (CIBPS). Descriptors of this syndrome are presented in Table 3. 


\section{Central psychological features}

Ramzy and Wallerstein (1958) have provided a psychoanalytic schematization of pain in man. The general sense of the points made by Ramzy and Wallerstein are cogent and in concordance with a vast clinical experience. Aside from the thorny issue of general psychoanalytic theory, their concepts are very useful in understanding how chronic pain readily can be regarded as 'real' pain in the absence of current nociceptive input. Their category of 'bodily pain' includes the presence and absence of current nociceptive input with a painful body locale identified. Their category of 'mental pain' includes dysphoric affect states of all types, usually without a body site located as painful. They have clearly divided pain into that that is readily consciously identified (with body locale) and pain that is largely subsumed in 'painful affects'. Also, they have a subcategory of 'neurotic bodily pain' that includes all bodily pain without discernible 'stimulus', and this category implies that memory mechanisms play a key role in this process. This is similar, if not identical, to our concept of pain without current nociceptive input. In their categorization, all the patients who fit in this category are characterized by them as having

TABLE 3. Chronic intractable benign pain syndrome

General characteristics

(1) Cannot be shown to be causally related to the here-and-now with any active pathophysiological or pathoanatomical process

(2) Has an antecedent history of generally ineffective medical and surgical intervention in the pain problem

(3) Has come to be accompanied by disturbed psychosocial function that includes the pain complaint and the epiphenomena that accompany it:

Epiphenomena

(1) Drug addiction of varying severity with attendant CNS side effects

(2) Multiple surgical procedures or pharmacological treatments with their own morbid side effects separate from those related to above

(3) Escalated decrease in physical functioning related to accompanying pain and/or fear that this pain is a signal of increased bodily harm and damage

(4) Escalated hopelessness and helplessness as persistent or increased dysphoria does not give way in the face of mounting numbers of 'newer' or different treatment interventions

(5) Emotional conflicts with medical care delivery personnel (doctors, nurses, therapists, technicians) that result in therapeutic goal interference

(6) Interpersonal emotional conflicts with significant others

(7) Escalated withdrawal and loss of gratifications from psychosocial activity

(8) Decrease in feelings of self-esteem, self-worth and selfconfidence

(9) Lasting, unpleasant mood and affect changes

(10) Decreased ability to obtain pleasure from the life process, reflected in the presence of profound demoralization and, at times, significant depression pain based on mainly hypochondriacal or hysterical mechanisms. We have described the chronic intrac- $\stackrel{2}{2}$ table benign pain syndrome in earlier publica-.. tions (Pinsky, 1975, 1978, 1983; Pinsky and Crue, $\overrightarrow{\vec{A}}$ 1984) and include Table 3 here in order to provide clinical descriptors for this syndrome in this discussion.

To pursue further the concepts of Ramzy and Wallerstein and the realities of the dynamic unity of mind and body (hence, psychosomatic), Pinsky is (1983) has used their general formulations in the $\overrightarrow{0}$ areas of 'affects' and 'ideas' and emphasized the strong input of cognitive factors. The dynamic interaction of many of these factors along with the non-nociceptive somatic input play the major role in chronic pain syndromes. The concept that nonnociceptive somatic stimuli may be 'perverted' in the central nervous system and be perceived as pain has been discussed above.

While there is no evidence to suspect any inherited or acquired defect in the central nervous system itself that leads to a chronic pain experience, there is the development of psychological forces (Table 2, no. 3) that have no known underlying neuroanatomical circuitry problems that play crucial roles in the appearance and maintenance of chronic pain syndromes. Psychological patterns of thought and be haviour, memory and fantasy can have significan roles in the development and the modulation of pain experience. General conditioning, operant corf ditioning, and social modelling can play significant roles in the shaping of some specific behaviours. However, the full syndrome generally seems to involve complex and multiple types of input to the learning process. There is a body of information that indicates that memories and attitudes about one's self formed in early life development have large affective colorations and play a very large role in character formation and subsequent abilities to acquire coping strategies that determine the types and levels of adaptation to the life process (Engel, 1959; Mersky, 1980; Meyer, 1980; Rado, 1980). These chronic pain syndromes seem to result from unconscious attempts to avoid, modify, or eliminate major problem life situations and patterns as well as affective states that can be of a highly complex nature.

When people develop these chronic intractable pain syndromes, we are confronted not only with pain behaviour (Fordyce, 1976) and abnormal illness $N$ behaviour (Mechanic, 1968; Pilowsky, 1969), but 0 with a more generalized maladaptive syndrome that $\mathbb{\sim}$ has multifaceted levels of disorder that are reflected $\underset{\omega}{N}$ in poor adaptation (Pinsky, 1983). In addition, our 우 psychological clinical experience with over $600 \mathrm{pa}-6$ tients with these types of chronic pain syndromes indicates that a large percentage have life histories $\stackrel{\mathscr{D}}{?}$ that include early childhood abuse (sexual and $\square$ 
physical), biological family alcoholism, and broken and multiple family existences. Serious questions about the relationships between depression as a disorder of mood and chronic pain syndromes remain unanswered (Pinsky, 1983; Sunde, 1983). Situational problems that are normally considered as contributing to psychological secondary gain are also commonly present. There is the as yet unanswered question about the extent to which premorbid psychological disorders play a role in the genesis of conflict situations that encompass the secondary gains that develop. From the aspect of classification, we believe that when there is any continued nociceptive input from underlying chronic pathophysiological conditions, the patients should be classified as having recurrent acute pain with psychological potentiation. Over time, if a patient's problem with pain becomes chronic (Table 1, nos. 5, 6) and there is no longer any evidence to invoke current nociceptive input, the classification of chronic pain is appropriate at that time. One can have recurrent acute pain to which psychological factors contribute greatly. The same can apply when a person suffers with pain related to cancer with ongoing nociceptive input from advancing pathology.

\section{Treatment and treatment outcome}

Placebo response in the treatment of patients with chronic centrally generated pain is one of the main reasons that we believe that results of treatment must adhere to five general steps (Table 4) in order to compare patient populations and their treatment outcome. The results of treatment of patients with chronic pain must attend mainly to long-term results before it can be determined if there were any beneficial effects of treatment (Crue, 1983). These are difficult to perform long-term follow-up studies, and we have reported on our work (Pinsky, 1983; Pinsky et al., 1979).

It must be pointed out again that all patients with chronic pain do not fulfill sufficient criteria for a diagnosis of a chronic intractable benign pain syndrome (CIBPS). As shown in Table 1, there are a number of patients under category 5 that continue to function reasonably well with chronic pain. There is no reliable epidemiological evidence that allows us to distinguish these two patient populations from one another (Crook, Rideout and Browne, 1984) except through observable behaviour and careful psychological evaluation that includes psychodynamic assessment. There are individuals whose coping abilities have not allowed for other adaptive responses to intrapsychic and interpersonal life events and may be prone to develop a chronic intractable benign pain syndrome. We belief that when these general underlying mechanisms are operative, they allow for classification (Table 2 as a combination of $3 a$ and $3 b$, 3c), as the so-called CIBPS syndrome (Pinsky, 1975, 1978, 1983; Pinsky and Crue, 1984; Pinsky et al., 1979).

\section{Conclusions}

Chronic pain is central 'neuralgic' pain which is psychosomatic in nature. The treatment for it should include considerations of the mechanisms involved in its development. Patients should not be subjected to useless and repetitious nerve block, or irreversible orthopaedic and neurosurgical procedures that can lead to procedure dependency, increased dysphoria, neurological deficits and increased suffering. Treatment should not include peripherally or centrally acting analgesic agents on a regular basis. When there is a depressive disorder of the type and magnitude that would be expected to respond to psychopharmacological or somatic treatment, this should be treated in the normal way.

Treatment for CIBPS should basically be psychotherapy, to enable somatic, intrapsychic, and life situational events to be interpreted and responded to by patients in ways that are less maladaptive. Because of the complex interplay of forces, the treatment programme needs to be sufficiently intensive and extensive. This can best be provided by a full inpatient or full-day treatment 4-5 days per week in a daycare treatment setting for 4-10 weeks. The treatment programme should be undertaken by treators trained in different disciplines working as a team in a medically-based setting (Crue, 1979). A full orientation to the patient is provided that simultaneously (not sequentially) attends to the medical (somatic) and psychological (intrapsychic, interpersonal, cognitive and behavioural) determinants of the pain.

\section{References}

Agnew, D.C., Crue, B.L. \& Pinsky, J.J. (1979) A taxonomy for diagnosis and information storage for patients with chronic pain. Bulletin of the Los Angeles Neurological Societies, 44, 84.

Carregal, E.J.A., Crue, B.L. \& Todd, E.M. (1963) Further observations of trigeminal potentials. Journal of Neurosurgery, 20, 277.

Crook, J., RIDEOUT, E. \& BRownE, G. (1984) The prevalance of pain complaints in a general population. Pain, 18, 299.

CRUE, B.L. (1979) A physiological view of the psychology of pain. Bulletin of the Los Angeles Neurological Societies, 44, 1.

CRUE, B.L. (ed.) (1979) Chronic Pain-Further Observations From City of Hope National Medical Center, Section V, Ch. 27-38. Spectrum Publications, New York.

CRUE, B.L. (1983) The centralist concept of chronic pain. Seminars in Neurology, 3, 331.

Crue, B.L. \& CARREgal, E.J.A. (1974) Postsynaptic repetitive neuron discharge in chronic neuralgic pain. Advances in Neuro$\log y, 4,643$.

Crue, B.L. \& Carregal, E.J.A. (1975) Pain begins in the dorsal horn-with a proposed classification of the primary senses. In: 
Pain, Research and Treatment. (Ed. B. L. Crue). Academic Press, New York.

Crue, B.L., Carregal, E.J.A. \& Todd, E.M. (1964) Neuralgiadiscussion of central mechanisms. Bulletin of the Los Angeles Neurological Societies, 29, 107.

Crue, B.L., Kilham, O., Carregal, E.J.A. \& Todd, E.M. (1967) Peripheral trigeminal potentials. Bulletin of the Los Angeles Neurological Societies, 32, 17.

Crue, B.L., Kenton, B. \& Carregal, E.J.A. (1976) Speculation concerning the possibility of a unitary peripheral cutaneous input system for pressure, hot-cold, and tissue damage: discussion of relationship to pain. Bulletin of the Los Angeles Neurological Societies, 41, 13.

Crue, B.L., Kenton, B., Carregal, E.J.A. \& Pinsky, J.J. (1980) The continuing crisis in pain research. In: Pain, Meaning and Management. (Eds. W. Smith, H. Merskey and S. Gross), p. I. Spectrum Publications, New York.

Crue, B.L. \& PinSKY, J.J. (1984) Different types of pain-with treatment considerations. Physician and Patient, (in press).

Crue, B.L., Pinsky, J.J., Agnew, D.C., Olial, R. \& Harth, L. (1979) Observations on the taxonomy problem in pain. In: Chronic Pain-Further Observations From City of Hope National Medical Center. (Ed. B. L. Crue), Ch. 2, p. 13. Spectrum Publications, New York.

Crue, B.L. \& Saltzberg, B. (1979) Dynamic pain. Bulletin of the Los Angeles Neurological Societies, 44, 127.

Crue, B.L., Shelden, C.H., Pudenz, R.H. \& Freshwater, D.B. (1956) Observations on the pain and trigger mechanism in trigeminal neuralgia. Neurology, 6, 196.

CRUE, B.L. \& TODD, E.M. (1968) Vagal neuralgia. In: Handbook of Clinical Neurology. (Eds. P. J. Vinken and G. W. Bruyn), Ch. 35, p. 362. North-Holland Publishing Company, Amsterdam.

Crue, B.L., Todd, E.M. \& Carregal, E.J.A. (1968) Cranial neuralgia. In: Handbook of Clinical Neurology. (Eds. P. J. Vinken and G. W. Bruyn), Ch. 27, North-Holland Publishing Company, Amsterdam.

Crue, B.L., TodD, E.M. \& CARregal, E.J.A. (1970) Observations on the present status of the compression procedure in trigeminal neuralgia. In: Pain and Suffering-Selected Aspects. (Ed. B. L. Crue), p. 47. Charles C. Thomas, Springfield, Illinois.

ENGEL, G.L. (1959) 'Psychogenic' pain and the pain-prone patient. American Journal of Medicine, 26, 899.

FELSÖÖRY, A. \& CRUE, B.L. (1976) Results of 19 years experience with sacral rhizotomy for perianal an perineal cancer pain. Pain, 2,431 .

FORDYCE, W.E. (1976) Behavioral Methods in Chronic Pain and Illness. C. V. Mosby, St Louis.

KAMDAR, M. (1979) Role of nerve blocks in the management of cancer pain. In: Chronic Pain-Further Observations From City of Hope National Medical Center. (Ed. B. L. Crue), p. 249. Spectrumळ Publications, New York.

Kenton, B., Crue, B.L. \& Carregal, E.J.A. (1975) Quantitativemeasures of the thermal reactivity of cutaneous mechanorecep- $\overline{\bar{D}}$ tors. Neuroscience Letters, 1, 321

Kenton, B., Crue, B.L. \& Carregal, E.J.A. (1976) The role of cutaneous mechanoreceptors in thermal sensation and pain. Pain, $2,119$.

MECHANIC, D. (1968) Medical sociology: a selective view. Free Press, New York.

MERSKey, H. (1980) In: Pain-Meaning and Management. (Eds. S. ڤ) L. Smith, H. Merskey and S. C. Gross). Spectrum Publications, New York.

MEYER, A. (1980) In: Comprehensive Textbook of Psychiatry/III(Eds. H. I. Kaplan, A. M. Freedman and B. J. Sadock), Vol. 1.3rdw edn. p. 809. Williams \& Wilkins, Baltimore.

PILOWSKY, I. (1969) Abnormal illness behaviour. British Journal of Medical Psychology, 42, 347.

PINSKY, J.J. (1975) Psychodynamics and psychotherapy in the treatment of patients with chronic intractable pain. Pain, Research and Treatment. (Ed. B. L. Crue), Ch. 34, p. 383. Academic Press, New York.

PINSKY, J.J. (1978) Chronic, intractable, benign pain: a syndromeo and its treatment with intensive short-term group psychotherapy Journal of Human Stress, 4, 17.

PINSKY, J.J. (1983) Psychodynamic understanding and treatment of the chronic intractable benign pain syndrome-treatment out- come. Seminars in Neurology, 3, 346.

PINSKY, J.J. \& CRUE, B.L. (1984) Intensive group psychotherapy with patients who have chronic intractable benign pain syn-ه dromes: operative factors. In: Textbook of Pain. (Eds. P. D. Wall and R. Melzack). Churchill Livingstone, London.

PINSKY, J.J., GRIFFIN, S.E., AGNEW, D.C., KAMDAR, M.D., CRIf , B.L. \& PINSKY, L.H. (1979) Aspects of long-term evaluationgfeo pain unit treatment program for patients with chronic intractafte benign pain syndrome: treatment outcome. Bulletin of the Angeles Neurological Societies, 44, 53.

Rado, S. (1980) In: Comprehensive Textbook of Psychiatry/III, Vol I. (Eds. H. I. Kaplan, A. M. Freedman and B. J. Sadock), 3rd edn., p. 777. Williams \& Wilkins, Baltimore.

RAMZY, I. \& WALlERSTEIN, R.S. (1958) Pain, fear and anxiety. Psychoanalytic Study of the Child, 13, 147.

Sternbach, R.A. (1974) Pain Patients: Traits and Treatment. Academic Press, New York.

ZBORowsKI, M. (1969) People in Pain. Jossey-Bass Inc., Publishers, San Francisco. 